\title{
Análisis de la Eficiencia Técnica Relativa de la Agroindustria en la Región de Tacna
}

\section{Analysis of the Relative Efficiency of the Technical Agroindustry in the Region of Tacna}

${ }^{1}$ Alcido Escobar Maquera

\begin{abstract}
RESUMEN:
Este estudio se realiza porque en la Región Tacna, no se tiene conocimiento sobre la eficiencia del trabajo y el capital en los diferentes eslabones de la cadena agroindustrial del orégano y el olivo como parte de su análisis económico. La hipótesis de trabajo es: que en el eslabón de la producción al menos un $30 \%$ de agricultores muestran niveles de eficiencia técnica cercanos a 1. El objetivo es medir la eficiencia del manejo de estos factores en el eslabón de la producción. Se eligió a la zona alto andina (Borogueña) donde se observó y encuestó a 109 productores de orégano, y a 49 de olivo en la costa (Magollo). El análisis se realizó con la estadística descriptiva. Los resultados muestran alta eficiencia en el trabajo 1,8\% (0,81-1,0) para el orégano; y el 6,1\%(0,86-1,0) para el olivo. Mientras en el capital es el $2,7 \%(0,81-1,0)$ para el orégano, y el 2,0\% $(0,85-1,0)$ para el olivo. Por lo tanto no se corrobora la hipótesis de trabajo.
\end{abstract}

Palabras Clave: Eficiencia Técnica Relativa, cadena agroindustrial

\begin{abstract}
:
This study is being carried out because of the fact that in the region of Tacna, there is no knowledge about the efficiency of labor and capital in the different links in the agricultural and industrial chain of the oregano and the olive tree as part of its economic analysis. The hypothesis is: that in the link of the production at least $30 \%$ of farmers show levels of technical efficiency close to 1. The objective is to measure the efficiency of the management of these factors in the link of the production. The high Andean zone (Borogueña) was chosen where 109 producers of oregano were observed surveyed, and 49 of the olive in the coast (Magollo). The analysis was conducted with the descriptive statistics. The results show high efficiency in the work $1.8 \%(0.81-1.0)$ for oregano; and $6.1 \%(0.86-1.0)$ for olive. While in the capital is $2.7 \%(0.81-1.0)$ for oregano, and $2.0 \%(0.85-1.0)$ for olive. Therefore it is false the hypothesis.
\end{abstract}

Key Words: Technical Efficiency Relative, agro-industrial chain. 


\section{INTRODUCCIÓN:}

Actualmente se reconoce que el desarrollo de la agroindustria es una alternativa con beneficios potenciales para el Perú. Mediante su desarrollo se logra integrar la agricultura con el sector industrial y el sector externo. La agroindustria como tal fue acuñado por Davis y Goldberg en 1957, definiéndolo como; la suma de todas las operaciones relacionadas al procesamiento y la distribución de insumos para la agricultura, operaciones de producción en las unidades agropecuarias y almacenamiento, procesamiento y distribución de los productos agropecuarios y sus bienes derivados, (Cannock y Gonzales, 1995). En este contexto, la medida de la eficiencia es un concepto clave que está relacionado directamente con la medida de la productividad. Sin embargo, no se trata de nociones análogas, aunque en ocasiones se emplean como sinónimos, principalmente cuando el interés del estudio se centra en comparar el rendimiento de las empresas (Distefano y Alderete, 2004). La productividad se define como la relación entre productos obtenidos y los insumos o recursos empleados; esto es, la cantidad de output obtenida por unidad de input empleada en el proceso de producción, (Grosskopf, 1993), asimismo, consiste en aumentar la calidad de los productos, mejorar la tecnología, la eficiencia en la producción, competir con éxito en segmentos de mercado cada vez más sofisticados e incluso competir en industrias totalmente nuevas y eficientes, todo lo cual, se expresa finalmente, en el valor del producto generado por unidad de trabajo o capital (Porter, 1990). Este tipo de eficiencia en la cual intervienen solamente magnitudes físicas de los insumos y productos, se conoce como eficiencia técnica, (Visconti, 2001). Pero el objetivo de este trabajo, se orienta más a la eficiencia relativa comparativa de distintas unidades económicas rurales en sus salidas y en sus entradas, con abstracción del proceso tecnológico y del entorno. De este modo se estará en la línea que proponía Farrell (1957) sobre la medición de eficiencia sosteniendo que "es imprescindible saber que tan lejos una industria puede esperar incrementar sus outputs solamente mediante un mejoramiento de la eficiencia", (Estellita y soares, 2004); (Banker et al, 1984).

Por otro lado, la agroindustria en el eslabón de la producción agrícola propiamente dicha usa como inputs a la tierra, capital y trabajo. La tierra no solamente es la superficie terrestre, sino también todas las características físicas y todo el ambiente natural que puede influir sobre la capacidad de la tierra para generar un producto. Con frecuencia encontramos el trabajo y la capacidad empresarial en una sola persona, en particular, en el propietario de un negocio, reservaremos para el trabajo el acto físico de realizar una tarea; y la capacidad empresarial la única responsabilidad de tomar decisiones. En el grupo restante, capital, asignaremos cualquier cosa hecho por el hombre, que se pueda utilizar como ayuda para aumentar la producción, (Bandini, 1982).

La agroindustria es una de las actividades de importancia económica y social de la Región Tacna, debido a su articulación "hacia atrás" y "hacia adelante".
Los problemas más importantes que debe afrontar el sector agroindustrial es la falta de aprovechamiento eficiente del mismo sector, en cuanto al uso de los insumos utilizados. Consecuentemente, la pregunta de investigación se centra en: ¿Cómo es el nivel de eficiencia técnica de la agroindustria en el eslabón de la producción de los productos bandera del orégano y el olivo?

\section{Hipótesis de trabajo}

La proposición planteada es: que en el eslabón de la producción del orégano y el olivo, al menos un $30 \%$ muestran niveles de eficiencia técnica en el uso de la mano de obra y del capital, que tienen ratios con valores cercanos o iguales a 1 (uno), los que pueden permitir dinamizar con satisfacción al eslabón del procesamiento.

\section{Objetivo}

Medir la eficiencia con las que se manejan los factores productivos de la mano de obra y del capital, en el eslabón de la cadena agroindustrial denominado "producción agrícola propiamente dicha".

\section{METODOLOGÍA}

Se recopilaron los datos mediante una encuesta estructurada durante los meses de setiembre y octubre del 2011. En el caso del orégano se eligió al distrito de Borogueña (Provincia Jorge Basadre), y para el caso del olivo a la Irrigación San Isidro de Magollo (Provincia Tacna). El tamaño de muestra fue calculado usando las fórmulas sugeridas por Hernández et al (2006), determinándose para el caso del Distrito de Borogueña 109 productores de orégano, y para el caso de Magollo 49 productores de olivo. Se utilizó el método paramétrico por intermedio del análisis estocástico de frontera, en donde el análisis se centró en el uso de la estadistica descriptiva.

\section{RESULTADOS}

Eficiencia Técnica Relativa de la Mano de Obra en el orégano.

En la Tabla Nro.1, muestra la eficiencia técnica relativa de la mano de obra, es decir para este caso consideramos que la frontera de producción máxima estaría representada por el valor 1; y para el ratio serían los valores cercanos a 1, entonces sólo se encontrarían el $1,8 \%$ de los productores con esta característica (entre $0,81$ y 1,00$)$, y los que son menores a 0,81 estarían en la ineficiencia, y corresponden al 98,2\%. Ver Fig. 1 .

Tabla Nro.1 Ratio: Eficiencia Técnica Relativa del Uso de la Mano de Obra

\begin{tabular}{|c|c|c|c|c|c|}
\hline & & Frecuencia & Porcentaie & Porcentaie vâtido & $\begin{array}{l}\text { Porcentaje } \\
\text { acumulado }\end{array}$ \\
\hline \multirow[t]{9}{*}{ Válidos } & $0.15<=X<0.26$ & 11 & 10.1 & 10.1 & 10.1 \\
\hline & $0.26<=X<0.37$ & 32 & 29.4 & 29.4 & 39.4 \\
\hline & $0.37<=X<0.48$ & 20 & 18.3 & 18.3 & 57.8 \\
\hline & $0.48<x<<0.59$ & 15 & 13.8 & 13.8 & 71.6 \\
\hline & $0.59<=X<0.70$ & 26 & 23.9 & 23.9 & 95,4 \\
\hline & $0.70<=X<0.81$ & 3 & 2.8 & 2.8 & 98.2 \\
\hline & $0.81<=x<0.92$ & 1 & 0.9 & 0.9 & 99.1 \\
\hline & $0.92<=X<=1.00$ & 1 & 0,9 & 0.9 & 100.0 \\
\hline & Total & 109 & 100.0 & 100.0 & \\
\hline
\end{tabular}

Fuente: Encuesta a productores de orégano, 2011 Elaboración: Propia 


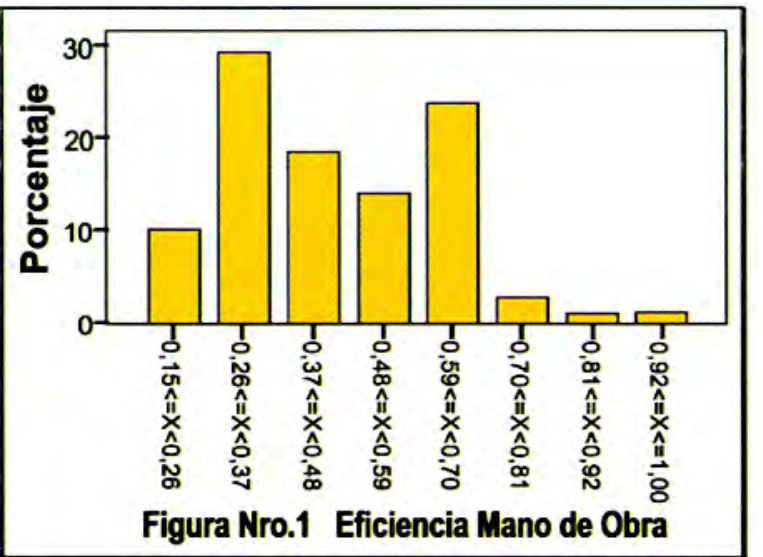

Eficiencia Técnica Relativa del Uso de Capital en el orégano

En el caso de la eficiencia técnica relativa del uso del capital ocurre algo similar a lo descrito para el caso de la mano de obra. La Tabla Nro. 2, revela que valores cercanos a 1 , sólo estarían el $2,7 \%$ que oscilan entre 0,81 y 1,00 y el resto que corresponde al $97,3 \%$ estarían en la ineficiencia debido a que sus valores están por debajo de 0,81 . Ver Figura Nro.2.

Tabla Nro. 2 Ratio: Eficiencia Técnica Relativa del Uso de Capital

\begin{tabular}{|c|c|c|c|c|c|}
\hline & & Frecuencia & Porcentaie & Porcertaie valido & $\begin{array}{l}\text { Poroentaje } \\
\text { acmuado }\end{array}$ \\
\hline \multirow[t]{9}{*}{ Válidas } & $0.15<x<0.26$ & 9 & 8.3 & \begin{tabular}{|c|}
8.3 \\
\end{tabular} & 8.3 \\
\hline & $0.26 c x<0.37$ & 31 & 28,4 & 28.4 & 36.7 \\
\hline & $0.37<x<0.48$ & 22 & 20.2 & 20.2 & 56,9 \\
\hline & $0.48 \Leftrightarrow x<0.59$ & 19 & 17.4 & 17.4 & 74.3 \\
\hline & $0,59 c=X<0,70$ & 21 & 19.3 & 19.3 & 93.6 \\
\hline & $0.70<x<0.81$ & 4 & 3.7 & 3.7 & 97,2 \\
\hline & $0.81<x<0.92$ & 2 & 1.8 & 1,8 & 99,1 \\
\hline & $0.92<x<=1,00$ & 1 & 0,9 & 0.9 & 100,0 \\
\hline & Total & 109 & 100.0 & 100.0 & \\
\hline
\end{tabular}

Fuente: Encuesta a productores de orégano, 2011 Elaboración: Propia

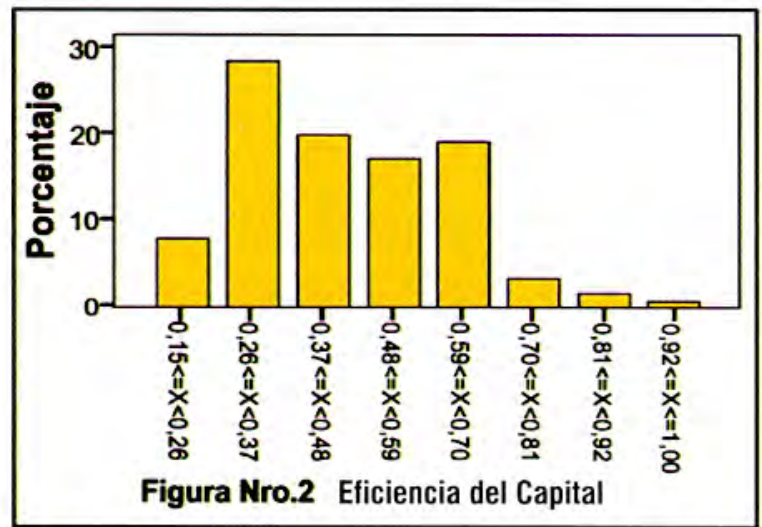

Eficiencia Técnica Relativa de la Mano de Obra en el olivo

La eficiencia relativa comparativa de distintas unidades económicas rurales, con abstracción del proceso tecnológico y del entorno, para el caso de la mano de obra de la agroindustria del olivo, dan cuenta de acuerdo a la Tabla Nro. 3, que existe un $6,1 \%$ que estaría ubicado en la frontera eficiente, dando como resultado el ratio entre 0,86 y 1,00 . Los demás quedarían como unidades económicas ineficientes, ya que exhiben ratios de eficiencia menores a 0,86 correspondiendo al $93,9 \%$.

Tabla Nro. 3 Ratio: Eficiencia Técnica Relativa del Uso de la Mano de Obra - Olivo

\begin{tabular}{|ll|c|c|c|c|}
\hline & Frecuencia & Porcentaie & $\begin{array}{c}\text { Porcentaje } \\
\text { váido }\end{array}$ & $\begin{array}{c}\text { Porcentaje } \\
\text { acumulado }\end{array}$ \\
\hline Válidos & $0,02<=\mathrm{X}<0.16$ & 18 & 36,0 & 36,7 & 36,7 \\
& $0.16<=\mathrm{X}<0.30$ & 17 & 34.0 & 34.7 & 71.4 \\
$0.30<=\mathrm{X}<0.44$ & 5 & 10.0 & 10.2 & 81.6 \\
$0.44<=\mathrm{X}<0.58$ & 4 & 8.0 & 8.2 & 89.8 \\
$0.58<=\mathrm{X}<0.72$ & 1 & 2.0 & 2.0 & 91.8 \\
$0.72<=\mathrm{X}<0.86$ & 1 & 2.0 & 2.0 & 96.9 \\
$0.86<=\mathrm{X}<=1.00$ & 3 & 6.0 & 6.1 & 100.0 \\
Total & 49 & 100.0 & 100.0 & \\
\hline
\end{tabular}

Fuente: Encuesta a productores de olivio, 2011 Elaboración: Propia

Lo que habría que advertir también es que existe una proporción importante de más baja eficiencia, $71,4 \%$ que su valor está entre 0,02 y 0,3 cifra lejana al referente identificado anteriormente $(0,86$ a 1,00$)$. Ver Figura Nro.. 3 .

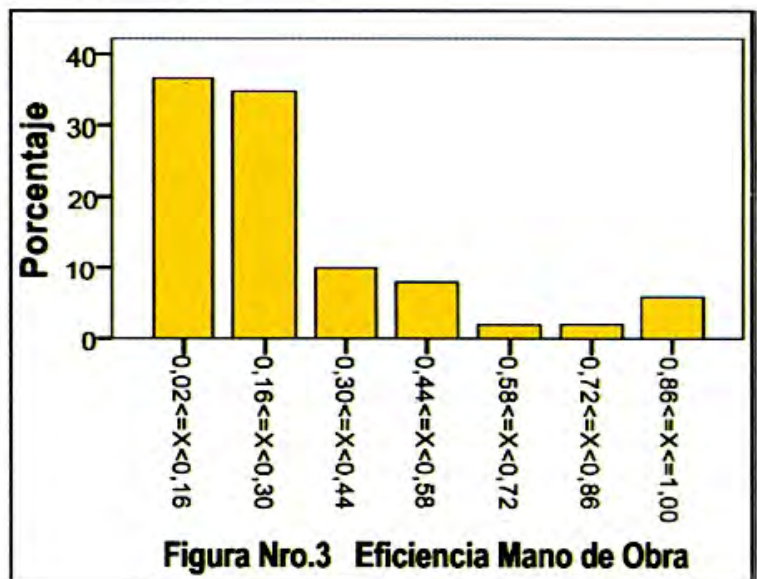

\section{Eficiencia Técnica Relativa del Capital en el olivo}

En la producción del olivo, la eficiencia técnica relativa del uso de capital, sólo existe un $2 \%$ que reporta un ratio entre 0,85 y 1,00 . Ubicándose el $98 \%$ restante por debajo de este valor; significando entonces una ineficiencia bastante marcada. Ver Tabla Nro. 4.

Tabla Nro. 4 Ratio: Eficiencia Técnica Relativa del Uso de Capital - Olivo

\begin{tabular}{|c|c|c|c|c|c|}
\hline & & Frecuencia & Porcentaie & Porcentaie valido & $\begin{array}{l}\text { Porcenteaje } \\
\text { acumulado }\end{array}$ \\
\hline \multirow[t]{5}{*}{ Válidos } & $0.01 \Leftrightarrow=x<0.15$ & 40 & 80.0 & \begin{tabular}{|l|}
81.6 \\
\end{tabular} & 816 \\
\hline & $0.15 \Leftrightarrow=X<0.29$ & 7 & 14.0 & 14.3 & 95.9 \\
\hline & $0.29=x<0.43$ & 1 & 2.0 & 2.0 & 98.0 \\
\hline & $0.85<=x<-1.00$ & 1 & 2.0 & 2.0 & 100.0 \\
\hline & Total & 49 & 100,0 & 100.0 & \\
\hline
\end{tabular}

Fuente: Encuesta a productores de olivo, 2011 Elaboración: Propia

Asimismo, en la tabla anterior, observamos una proporción de mayor magnitud $81,6 \%$ que son los que tienen la más baja eficiencia reportando un valor de entre 0,01 y menos de 0,15 por demás alejado del referente indicado como parte de la frontera eficiente. Ver Figura Nro. 4 


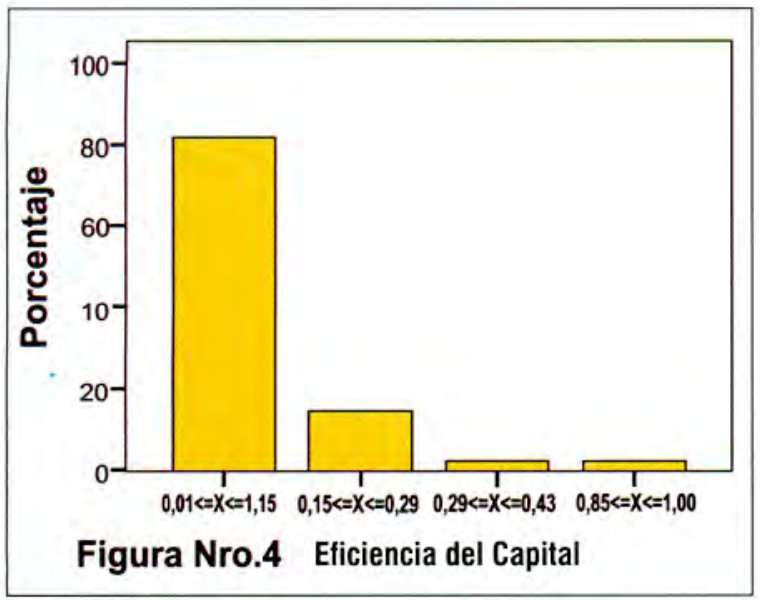

\section{DISCUSIÓN}

Los rendimientos por hectárea en el orégano están entre 997 y $5215 \mathrm{~kg} / \mathrm{ha}$ de orégano seco. Obviamente estos rendimientos sobre todo para $5215 \mathrm{~kg} / \mathrm{ha}$ no es fácil obtenerla, debido a que la mayor parte de los que producen son pequeños agricultores, en el que se dificulta implementar tecnologías aparte de ser caras, así como por su idiosincrasia que no les permite adoptar nuevas tecnologías. Sumado a ello claro está el uso intensivo de la mano de obra ( 35 a 218 jornales/ha) en comparación con el olivo desde su rendimiento que va de 600 a $14000 \mathrm{~kg} / \mathrm{ha}$, que por la ubicación podrían tranquilamente alcanzarse a la frontera eficiente. Es más al contrastarse con el orégano para el aspecto de la mano de obra se observa que el olivo utiliza menos ( 2 a 44 jornales/ha).

En relación al factor capital comparando ambos cultivos pareciera que no habría diferencia significativa, dado a que en el orégano se reporta inversiones entre S/. 1066 y $\mathrm{S} / .8310$ y para el caso del olivo se tiene entre $\mathrm{S} / .259$ y S/.10161 por hectárea.

En cuanto a las productividades de la mano de obra y de capital en ambos cultivos agroindustriales, notamos que en el orégano el $0,9 \%$ de los productores tiene una productividad de 59 a $67 \mathrm{~kg}$ y el $7,3 \%$ obtiene la productividad más baja entre 10 y menor a $17 \mathrm{~kg}$. Probablemente se deba a que necesariamente deba ir acompañada por la tecnología en términos de insumos que ayuden al desarrollo del cultivo y su producción. En cambio en el olivo el $6,1 \%$ de los productores alcanza una productividad entre 1289 y $1500 \mathrm{~kg}$, y la más baja $28,6 \%$ entre 23 y menores a $234 \mathrm{~kg}$. Sobre este aspecto debe señalarse que la zona donde se estudió fue San Isidro de Magollo, en donde parece que existe un proceso de sustitución de cultivos, es decir se dedican más a la vid que al olivo, motivo por lo que acusan bajas producciones porque también usan bajos insumos.

El capital usado en el orégano el 1,8\% de los productores tiene una productividad alta de $1,71 \mathrm{~kg}$ a $2,16 \mathrm{~kg}$, en cambio el 7,3\% tiene una productividad entre $0,33 \mathrm{~kg}$ y menor a $0,56 \mathrm{~kg}$, esto se debe como dijimos anteriormente al poco uso de insumos modernos y tecnología. Igual situación parece ocurrir en el caso del olivo, cuando se reporta que sólo el $2 \%$ tiene una productividad de capital de $18,08 \mathrm{~kg}$ y $21,02 \mathrm{~kg}$, y lo más bajo reportado lo alberga el $81,6 \%$ de los productores que tiene una productividad de capital entre $0,26 \mathrm{~kg}$ y $3,23 \mathrm{~kg}$.

De lo descrito anteriormente podemos decir que los ratios de eficiencia técnica relativa en el uso de la mano de obra $1,8 \%$ que tiene el valor entre 0,81 y 1,00 para el orégano, y $2 \%$ que tiene el valor entre 0,86 y 1,00 para el caso del olivo, nos hacen concluir que el resto se califican como ineficientes. La explicación podría decirse a nivel de hipótesis que no hayan llegado los servicios de extensión y transferencia tecnológica o si lo han hecho simplemente no han sido adoptadas. Por otra parte puede ser que existan problemas de organización, y finalmente porque su racionalidad no les permite superar inconvenientes que tienen que ver con la adopción de tecnologías. Igualmente para el caso de la eficiencia técnica relativa del uso de capital, se tiene encontrado para el caso del orégano que el $2,7 \%$ de los productores alcanza un valor entre 0,81 y 1,00 ; y para el olivo el $2 \%$ muestra valores entre 0,85 y 1,00 siendo el resto de los productores catalogados como ineficientes. Estos resultados también pueden deberse a las razones comentadas.

\section{CONCLUSIONES}

La eficiencia técnica relativa de la agroindustria del orégano alcanzada en la mano de obra, sólo lo constituye el $1,8 \%$ de los productores con valores de 0,81 a $1,00 \mathrm{y}$ el $98,2 \%$ exhiben valores menores a 0,81 por consiguiente estarían en la ineficiencia. Por el lado del uso del capital el $2,7 \%$ de los productores son eficientes por mostrar valores que oscilan entre 0,81 y 1,00 y $97,3 \%$ son ineficientes debido a que sus valores están por debajo de 0,81 .

En la agroindustria del olivo, el 6,1\% de los productores se ubican en la frontera eficiente en el uso de la mano de obra, y que presenta ratios entre 0,86 y 1,00. Los demás $(93,9 \%$ de productores) quedarían como unidades económicas ineficientes, ya que exhiben ratios a 0,86 . En el uso del capital sólo existe un $2 \%$ de productores eficientes que reporta un ratio entre 0,85 y 1,00 mientras que el $98 \%$ restante es ineficiente porque sus valores son menores a 0,85 .

\section{REFERENCIAS BIBLIOGRÁFICAS}

ANDERSON, D. y SWEENEY, D. y WILLIAMS, T.: (2004): "Métodos cuantitativos para los negocios". Editorial International Thomson - México.

BANDINI MARIO (1982): "Economía Agraria". En: Diccionario de Economía Política. Edit. COCULSA. Madrid -España.

COOPER, W., SEIFORD, L. y TONE, K. (2000): "Análisis envolvente de datos" - 2a. Ed. Kluwer Academic Publishers, Massachusetts, USA. 
CANNOCK G. Y A. GONZÁLES (1995): "Economía Agraria”. Edit. Universidad del Pacífico. Lima-Perú

CRAMER G., Y C.JENSEN (1995): "Economía Agrícola y Agroempresas". Editorial Limusa - México.

DISTÉFANO, V. y ALDERETE, V. (2004): "La gestión a partir de la productividad - Medición y mejora en distintas organizaciones" - Trabajo presentado al XXVII Congreso Argentino de Profesores Universitarios de Costos - Tandil - Buenos Aires.

ESTELLITA LINS M, SOARES DE MELO JC, ANGULO MEZAE. (2004):

"Selección de Variables para el Incremento del Poder de Discriminación en los Modelos DEA ". Revista de la Escuela de Perfeccionamiento en Investigación Operativa, 24, pp. 40-52.

FARRELL N. J. (1957): "La medición de la eficiencia productiva". Revista de la Real Sociedad de Estadística. Serie A, vol 120,pp. 253-281.
GROSSKOPF, S. (1993): "La eficiencia y la productividad", en H. O. Frito, C. A. K. Lovell y S. S. Schmidt (eds.): La medición de eficiencia productiva. Técnicas y aplicaciones, Oxford University Press.

PORTER M. (1990): "Las ventajas competitivas de las Naciones". Artículo publicado en la Revista Comercio Exterior-México.

VISCONTI, R. (2001): "Costos, Productividad, Competitividad" - Trabajo presentado al XXIV Congreso Argentino de Profesores Universitarios de Costos-Córdoba.

\section{Correspondencia:}

Alcido Escobar Maquera

Ciudad Universitaria fundo "Los Granados" Av. Miraflores s/n. Tacna. Perú.

alesma13@hotmail.com 